\title{
Controlled fabrication of nickel perpendicular nanocontacts using focused ion beam milling
}

\author{
Hong-Xiang Wei \\ State Key Laboratory of Magnetism, Institute of Physics, Chinese Academy of Sciences, \\ Beijing 100080, China and Physics Department, Trinity College Dublin, Dublin, Ireland \\ R M. Langford \\ Physics Department, Trinity College Dublin, Dublin, Ireland \\ Xiufeng $\operatorname{Han}^{\mathrm{a})}$ \\ State Key Laboratory of Magnetism, Institute of Physics, Chinese Academy of Sciences, \\ Beijing 100080, China \\ J. M. D. Coey \\ Physics Department, Trinity College Dublin, Dublin, Ireland
}

(Presented on 1 November 2005; published online 17 April 2006)

\begin{abstract}
A method for the controlled fabrication of a nickel perpendicular nanocontact with diameters less than $5 \mathrm{~nm}$ is reported. The method involves milling pores through the $100 \mathrm{~nm}$ thick silicon nitride membrane using a focused ion beam, and depositing thin $\mathrm{Ni}$ film on the both side of the silicon nitride membrane. The shape of the resulting pore and nanocontact size is determined by the initial hole size and redeposition of the sputtered material onto the sidewalls of the holes. A sub-5 nm Ni nanocontact was prepared and the $I-V$ and $R-H$ characteristics measured. (C) 2006 American Institute of Physics. [DOI: 10.1063/1.2150389]
\end{abstract}

\section{INTRODUCTION}

Nanocontacts with dimensions of less than $20 \mathrm{~nm}$ have been fabricated using a variety of techniques. Nanoimprint lithography, ${ }^{1}$ using an atomic force microscopy tip, has been used to prepare sub $10 \mathrm{~nm}$ perpendicular contacts. Electrodeposition at the end of electrodes ${ }^{2}$ or nickel wires ${ }^{3}$ has been used to make planar ballistic nanocontacts. Focused ion beam (FIB) milling ${ }^{4}$ of UV and e-beam patterned structures has also been used to fabricate nanoconstrictions, but unfortunately, this method can result in gallium implantation and damage which alters the physical and structural properties ${ }^{5}$ of the sample. Here, a FIB based technique for the fabrication of perpendicular nanocontacts is outlined. The FIB is used to mill pores through a silicon nitride membrane upon which metals are then sputtered. It is shown that if suitable milling parameters are chosen (i.e., initial hole size and milling time) the technique, through redeposition on the sidewall of the pores, makes it possible to control the nanocontact size down to sub-5 $\mathrm{nm}$.

\section{EXPERIMENTAL SETUP}

Silicon nitride membranes were prepared following the procedures of Grant et al. and Enquist et al. ${ }^{6,7}$ For the FIB milling (FEI Dual Beam 235 system) a beam current of 10 $\mathrm{pA}$, a dwell time of $30 \mu \mathrm{s}$, a pixel spacing of $5 \mathrm{~nm}$, and an emission current of $2.2 \mu \mathrm{A}$ were routinely used. However the effect of changing these parameters and the thickness of the silicon nitride membranes on the size of the nanocontacts that were produced was also investigated.

\footnotetext{
${ }^{a)}$ Electronic mail: xfhan@aphy.iphy.ac.cn
}

To assess the effect of different starting hole sizes and doses/times on the pore size and shape an array of holes were milled. The shapes of the milled pores were determined by two methods: (i) by using scanning electron microscopy (SEM) to measure the hole sizes on both sides of the membrane and (ii) by FIB cross sectioning, SEM, and transmission electron microscopy (TEM) imaging. To enable the holes to be found when the membrane was reversed alignment crosses were milled. For cross sectioning, e-beam assisted deposition $(5 \mathrm{keV}, 1 \mathrm{nA})$ was exploited to deposit the protective Pt strap rather than using ion beam assisted deposition so that the pore shapes was not altered by possible milling by the ion beam. Furthermore, during analysis and milling, care was taken not to image the holes/pores with either the ion or electron beam. Ion imaging could increase/ alter the pore sizes/shapes while electron beam imaging could cause carbon, from the breakdown of hydrocarbons, to be deposited on the sidewalls of the pores and thus reduce their size.

The nanocontacts were then prepared by sputtering a 30 $\mathrm{nm}$ thick nickel film onto both sides of the silicon nitride membrane. To prevent shorting between the top and bottom electrode, copper tape was used to mask the sample's edges during sputtering.

\section{RESULTS AND DISCUSSION}

Figure 1(a) shows a cross-sectional SEM image of the sample prepared by FIB. It is found that if the size of the hole milled through the membrane is more than $150 \mathrm{~nm}$, the shape of the hole is almost cylindrical, as shown in Fig. 1(b). The holes become conical when the hole size is smaller than $150 \mathrm{~nm}$ and it is difficult to cut the small hole through the 

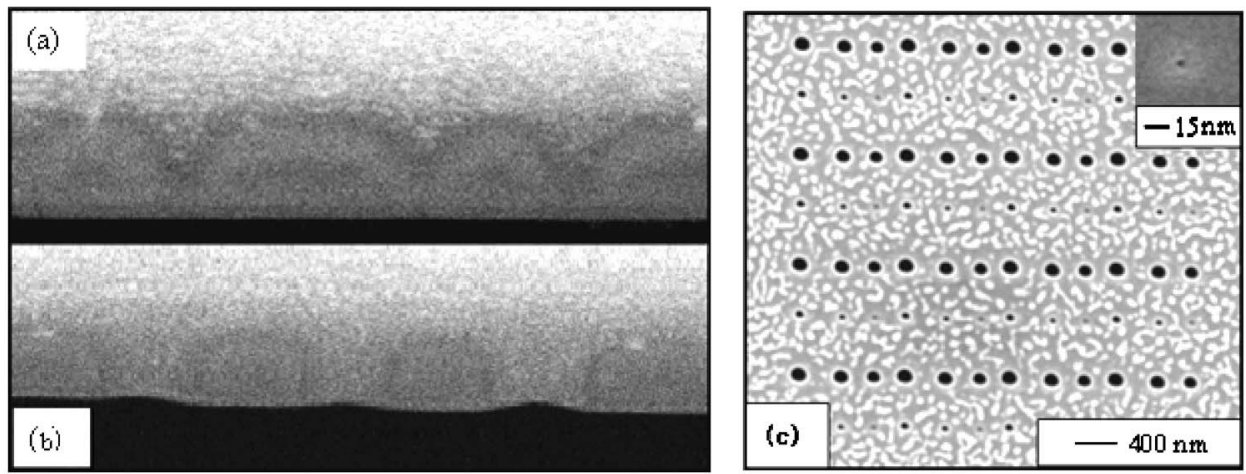

FIG. 1. SEM images of (a) and (b) a FIB milled conical shaped nanopore through a $100 \mathrm{~nm}$ thick silicon nitride membranes. (c) Typical holes viewed from the top and bottom surface of a $100 \mathrm{~nm}$ thick silicon membrane.
TABLE I. Hole diameters measured from both sides of the silicon nitride membranes.

\begin{tabular}{lccccccc}
\hline \hline & 200 & 200 & 150 & 100 & 60 & 40 & 30 \\
$\begin{array}{l}\text { Initial hole diameter (nm) } \\
\begin{array}{l}\text { Diameter measured on bottom } \\
\text { of membrane (nm) }\end{array}\end{array} 200$ & 200 & 145 & 80 & 30 & 10 & Sub. 5 \\
\begin{tabular}{l} 
Milling time (s) \\
\hline \hline
\end{tabular} & 0.7 & 0.5 & 0.5 & 0.4 & 0.4 & 0.4 & 0.3 \\
\hline
\end{tabular}
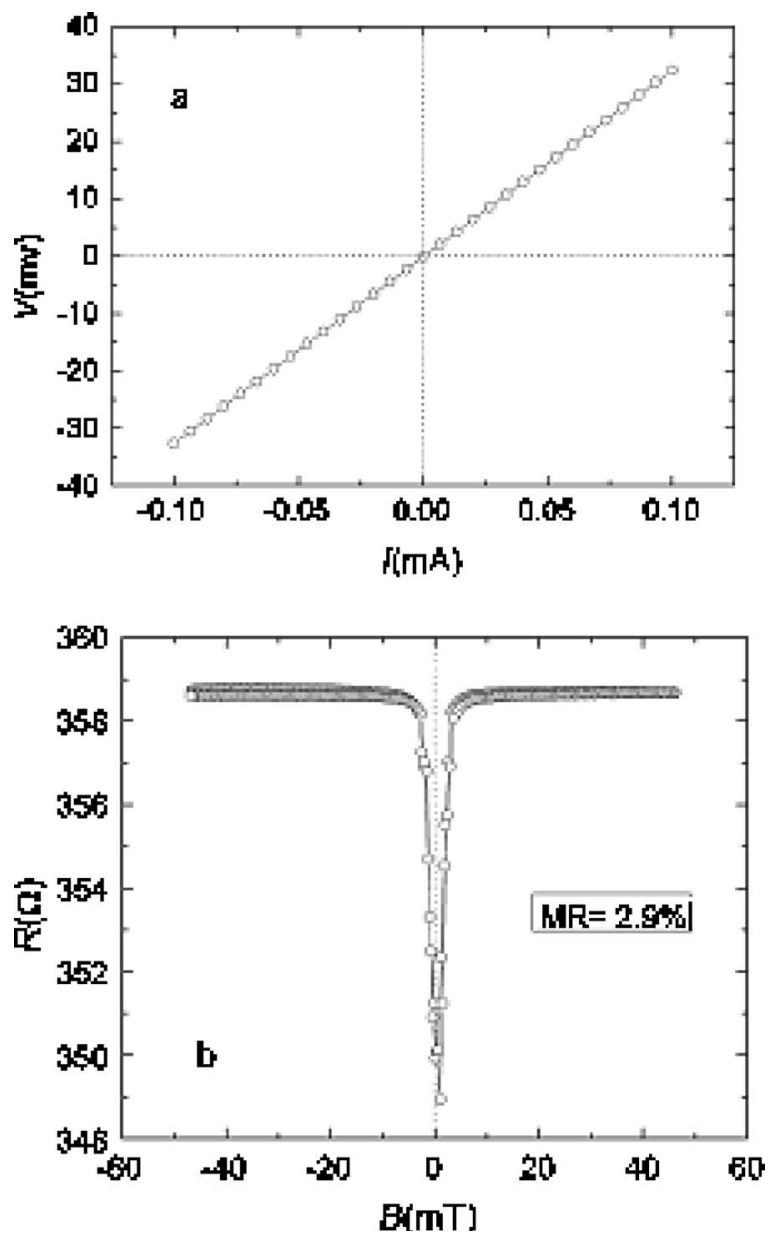

FIG. 2. (a) $I-V$ and (b) MR curve of a nickel nanocontact fabricated by milling through a silicon nitride membrane. center, perforating the membrane. Therefore, when the holes are cut it seems that they have not passed through the full thickness of the membrane only because of the small size, as shown in Fig. 1(b). Figure 1(c) shows the SEM images from the top side of the membrane. The diameter of the $40 \mathrm{~nm}$ hole reduced to sub- $3 \mathrm{~nm}$ at the bottom, as shown in the inset of Fig. 1(c). The diameters of some of the selected holes measured from the top and bottom sides of the membrane are given in Table I. It was observed that the pore shape and size passing through the membrane strongly depends on the milling time, regardless of the diameter of the initial hole.

The data in Table I indicate that if the appropriate milling parameters are chosen (initial hole size and milling time) then the size of the nanocontact can be controlled down to less than $5 \mathrm{~nm}$. The size of the nanocontact is determined by the amount of redeposition, which is determined by the aspect ratio of the pore (i.e. the initial hole size and membrane thickness). If the FIB column is aligned correctly and the beam current is stable then the technique is highly reproducible, enabling the sizes of the nanocontacts to be controlled.

Figures 2(a) and 2(b) show a typical $I-V$ curve and magnetoresistance (MR) curve for a nickel nanocontact prepared by the conical pore method. The magnetic field was applied perpendicular to the nanocontact, in the plane of the film. The MR value (3\%) shown was the largest value obtained for any of the nickel nanocontacts prepared in this way. The origin of this magnetoresistance is discussed elsewhere. From the resistance and using Sharvin's formula ${ }^{9,10}$ the size of the nanocontact is calculated to be $2 \mathrm{~nm}^{8}$ which agrees with the sizes determined by SEM and TEM.

\section{CONCLUSION}

A method for the controlled fabrication of a nickel perpendicular nanocontact has been outlined. It consists of milling pores into silicon nitride membranes and using the redeposition of the sputtered material to control the diameter of the nanocontact. The technique has been demonstrated by preparing Ni nanocontact with less than $5 \mathrm{~nm}$, which agrees with the Sharvin's formula. The largest MR observed was $3 \%$.

\section{ACKNOWLEDGMENTS}

The work was supported by the China-Ireland Science and Technology Collaboration Research Fund provided by Science Foundation Ireland (SFI) and the Chinese Ministry of Science and Technology. 
${ }^{1}$ J. Carrey, K. Bouzenehouane, J. M. George, C. Cenerey, T. Blon, M. Bides and A. Vaures, S. Fusil, S. Keanne, L. Villa, and L. Piraux, Appl. Phys. Lett. 81, 760 (2002).

${ }^{2}$ C. S. Lang, J. Thiltges. B. Doubin, and M. Johnson, J. Phys.: Condens. Matter 14, 1765 (2002).

${ }^{3}$ H. D. Chopra and S. Z. Hua, Phys. Rev. B 66, 020403 (2002).

${ }^{4}$ O. Cespedes, M. A. Bari, C. Dennis, J. J. Versluijs, G. Jan, J. O’Sullivan,

J. F. Gregg, and J. M. D. Coey, J. Magn. Magn. Mater. 242, 492 (2002)
${ }^{5}$ D. Ozkaya, R. M. Langford, L. Wai, and A. K. Petford-Long, J. Appl. Phys. 91, 9937 (2002).

${ }^{6}$ A. W. Grant, Q-H Hu, and B. Kasemo, Nanotechnology 15, 1175 (2000). ${ }^{7}$ Enquist $\mathrm{F}$ and Spetz A, Thin Solid Films 145, 99 (1986).

${ }^{8} \mathrm{H}$.-X. Wei et al. (unpublished).

${ }^{9}$ Yu. V. Sharvin, Zh. Eksp. Teor. Fiz. 48, 984 (1965).

${ }^{10}$ A. G. M. Jansen, A. P. van Gelder, and P. Wyder, J. Phys. C 13, 6073 (1980). 